\title{
Lump formation in Filtration and Drying of Particulate APIs
}

\author{
Ajinkya Pandit* and Gavin Walker \\ Bernal Institute, University of Limerick, Ireland \\ *Ajinkya.Pandit@ul.ie
}

\begin{abstract}
Crystallization, filtration and drying are important separation and purification unit operations in the pharmaceutical and speciality chemical industries. Continuous processing technologies have emerged as a promising alternative to the more conventional batch processing. Previous studies primarily focus on crystallization and as a result subsequent stage of filtration and drying are overlooked. In the present short communication, a phenomenological parallel is drawn with the drying of porous solids, studies for which are relatively widely reported. Such analysis helps to identify, the fundamental cause for lump formation, and possible mitigation strategies. Based on the postulated physical scenario, two prototype designs to intensify continuous operation of filtration and drying units are discussed. The prototype solutions could make it possible to intensify or combine multiple stages of filtration, deliquoring, washing and drying thus enabling efficient continuous processing of solid form pharmaceutical and specialty chemicals.
\end{abstract}

\section{Keywords}

drying, filtration, continuous processing, active pharmaceutical ingredients, pore network models 


\section{Introduction}

In drying, a system consisting of a stack of closely but randomly packed particles with the solvent filling the interstitial void between the particles is considered. During drying, it is expected for the air to completely displace the solvent filling the void. However, it is often not the case as some solution is still trapped - leading to the lumping. To cause this trapped solvent to evaporate, recently a method was tested by Ottoboni et. al. (2020) ${ }^{[1]}$ wherein hot air was used to displace and/or evaporate the solvent. A continuous filter dryer (CFD) setup developed by Alconbury Weston Limited (AWL) was used by Ottoboni et. al. (2020) ${ }^{[1]}$. The drying of the cake was achieved a criterion to detect the completion of drying, as a sudden rise in the outlet temperature, was developed. However, lumping (aggregation of particles to form a random continuous blocks) was still observed to various degrees in the dried samples depending upon the operating conditions as shown in Figure 1.

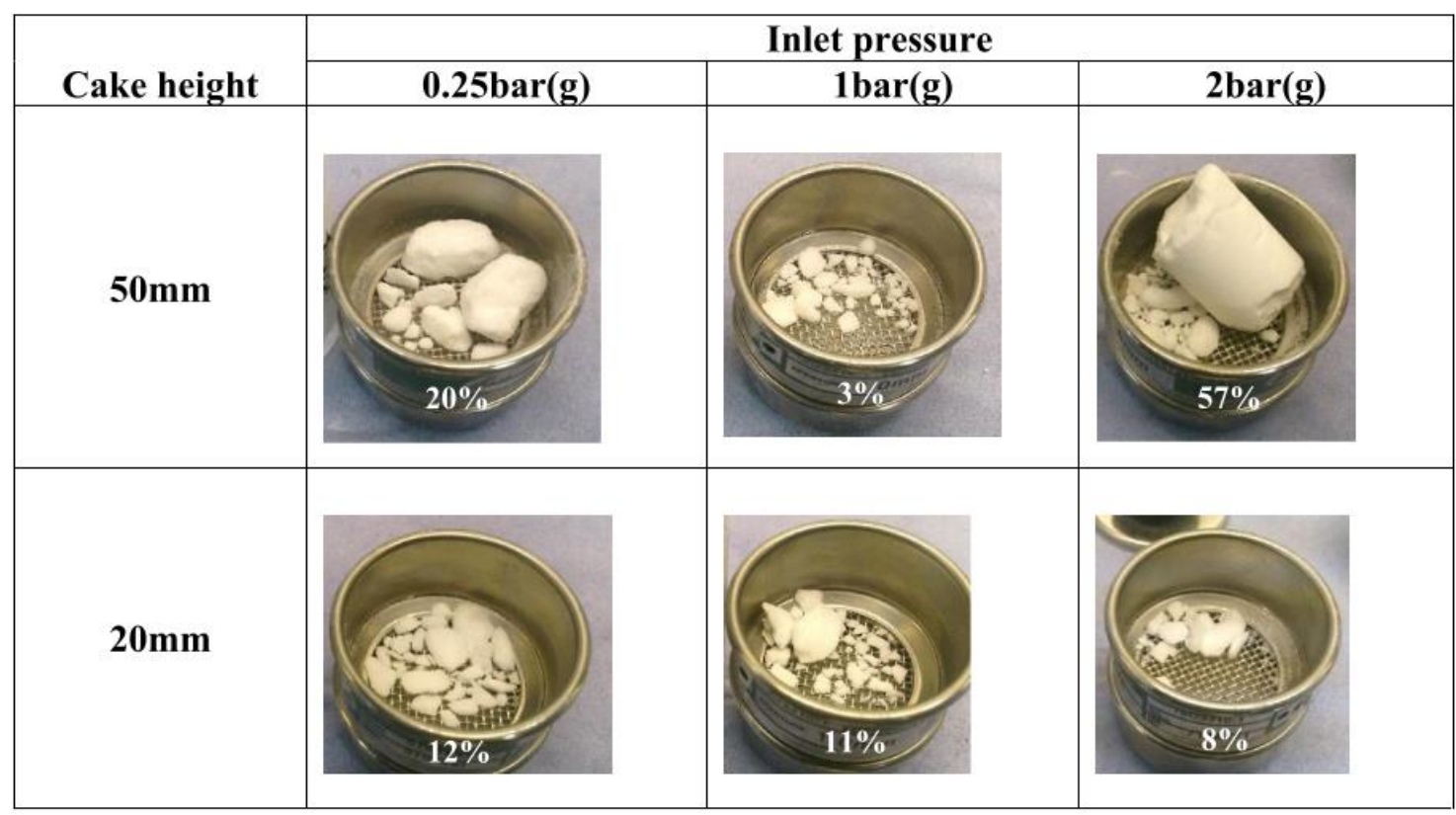

Table 1: Extent of agglomeration of sodium bicarbonate in water under different operating conditions. Percentage values denote the extent of agglomeration. (Figure reported by Ottoboni et. al., $2020^{[1]}$ and reproduced with permission from ACS on 21/07/21. Further permissions regarding the reuse of this material should be directed to the ACS)

Lumping or caking is not desirable the lumped portion of the powder is rendered useless for further processing, say for instance to make tablets etc. The mechanism of lumping or caking may be typically described as follows - the solution contained within the interstitial spaces is not completely displaced during the dewatering/drying process and over time becomes saturated with the solute due to indefinite contact. On being kept for longer drying times (for instance for 24 hours as was done by Ottoboni et. al., $2020^{[1]}$ ) allows the complete removal of the solution - through evaporation, leaving behind the dissolved solids to form lose interconnections between the particles leading to lumping. 
Hence, it was observed in the case of Ottoboni et. al. (2020) ${ }^{[1]}$, that passing the hot dry air did not completely solve the problem of lumping, especially during higher cake heights.

The process of completely removing the liquid from the cake is known as deliquoring. The conventional solution to this problem is to do two washes before deliquoring using the dry air. The first wash is done by using the crystallisation solvent itself to displace the saturated solvent and then the first wash is quickly displaced by adding a second wash solvent - which is both miscible with respect to wash solvent $A$ but also has a lower solubility for the solute. This has been reported to lower the extent of the solute precipitating out and thus decreasing the lumping ${ }^{[1-3]}$. However, lumping is still observed and especially may be expected to increase on further scale-up ${ }^{[3]}$.

\section{Pore Network Model}

Hence, it is useful to just step back and consider other approaches to the problem. In this regard, the pore network model (PNM) is typically used to study the removal of water content from porous solids. This subject has been extensively studied previously ${ }^{[4]}$ although the application of these models to the drying of particles after crystallisation/filtration is to the authors knowledge not present. Debaste et. al. (2010) ${ }^{[5]}$ studied the fluidized bed drying of yeast granules by combining the PNM with a fluidized bed model. Indeed, a solid cake may be equivalently considered as a porous solid - with a pore network representing the vacuous space typically wetted using a solvent. The first caveat here is that PNM studies this author has come across usually considers drying under stagnant air. The second caveat is that in PNM studies, typically only one surface is kept open to drying so the air does not 'pass through the solid'. However, on the positive side, the considered pore sizes (of the order of $10 \mathrm{~s}$ of microns) overlap very well with the particle sizes, and consequently the void sizes, typically expected after crystallization. Hence it is very useful to attempt to extrapolate key learnings from the PNM studies and apply them for the case of drying of solids.

Rather than going into the specifics of the mathematical description of the PNM, it is worthwhile to understand the PNM framework which provides very good fundamental insights with regards to the physics of the drying problem. A porous solid in a PNM is typically represented as a grid as shown in Figure 1. Figure 1 demonstrates a schematic representation of an experiment using a silicone micromodel saturated with a salt-water solution and with the top surface kept open for drying. For more details on the relevant experiment, the reader is urged to refer Metzger, $2019^{[4]}$. In our case, the solid squares which form the grid may be considered as the particles and the channel network may be considered as the vacuous network contained within the particles. This experiment is highly relevant as it not only gives an intuitive understanding of the progression of drying, but also demonstrates the deposition of solids leading to lumping. As the saturated solution slowly evaporates, 
it deposits solids within the voids thus forming interconnecting bridges. The severity of the deposition of solids within the voids depends on the solubility of the solute in the trapped liquid. Although this representation is a $2 \mathrm{D}$ picture, the principles should equally be valid while considering a 3D case, albeit be a bit harder to visualise.
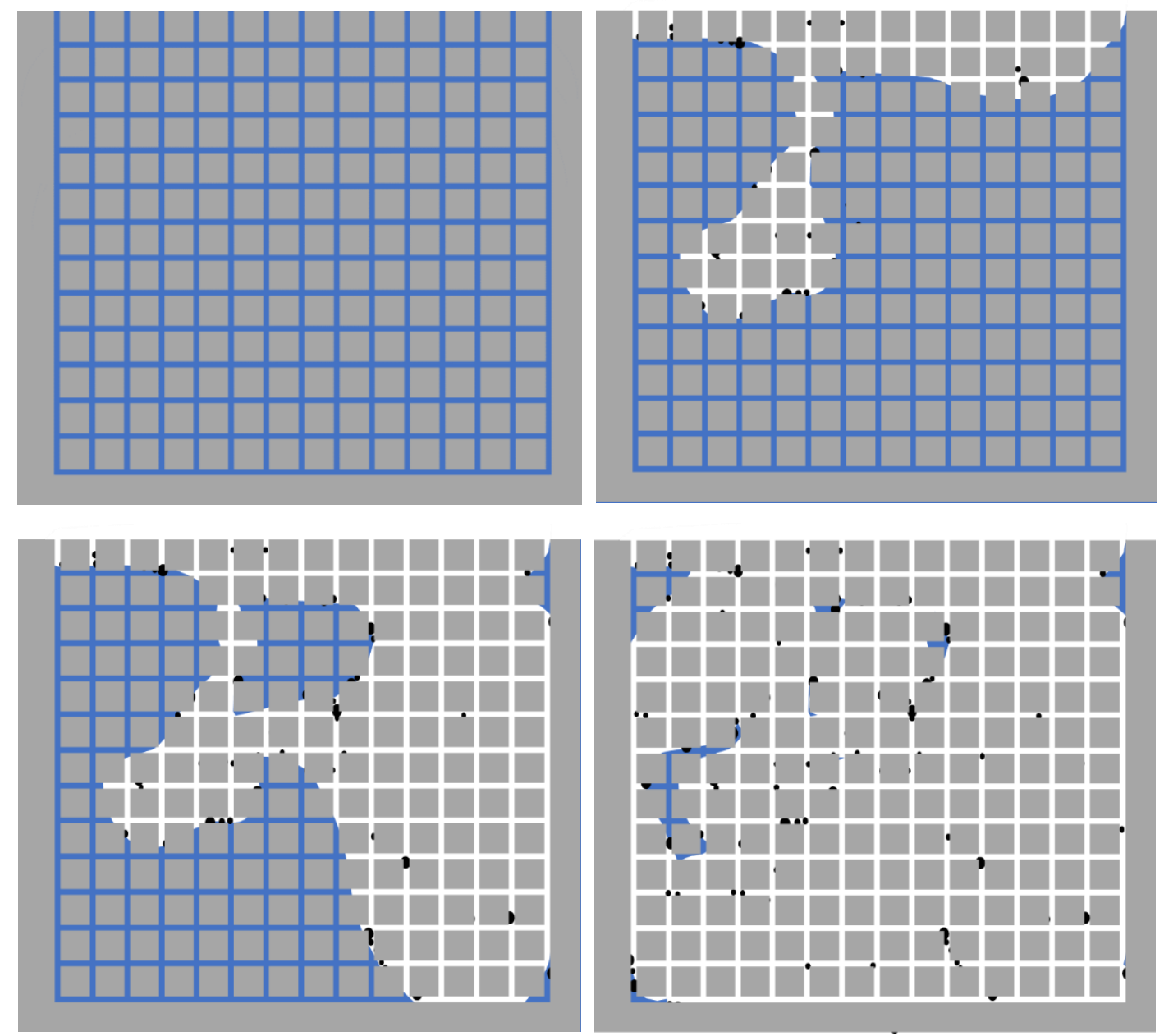

Figure 1: Qualitative representation of the progression of an experiment performed to investigate the drying of a porous solid using a silicone micromodel saturated with a salt-water solution

Several 3D simulations have also been performed, although the sheer number of pores/particles makes a complete solution intractable. Shown in Figure 2 are the results of an experiment performed wherein the drying of a fixed bed of mono-sized spherical glass particles was non-invasively tracked using X-ray tomography. Initially as shown in case (a), the entire liquid is almost filled to the brim. In the case (b), the liquid network at the wall was drained and visually the cake may be considered as dry but there is a significant amount of liquid trapped within the interstitial spaces. In this case, the drying is limited by the total surface area of the interface, which is very less (boundary of the liquid as shown in Figure 1) as compared to the total particle surface area. Finally, after extended drying duration liquid clustering is observed as shown in case (c) where the liquid continuum breaks up into 
disconnected clusters. This picture is entirely analogous as for the case of drying solids, with added complexities of possible non uniformity in terms of shape and size and the deposition of solids.

(a)

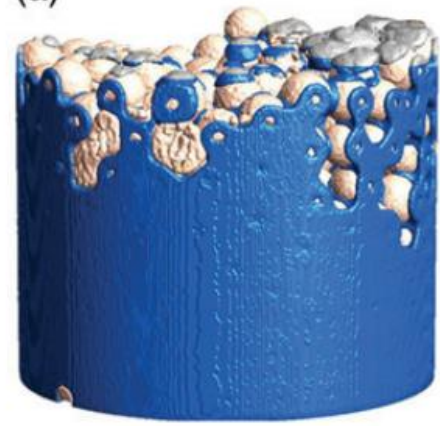

(b)

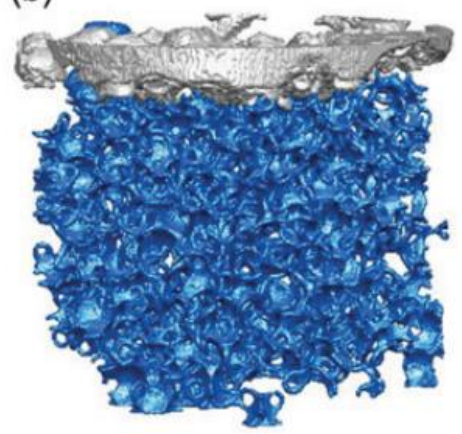

(c)

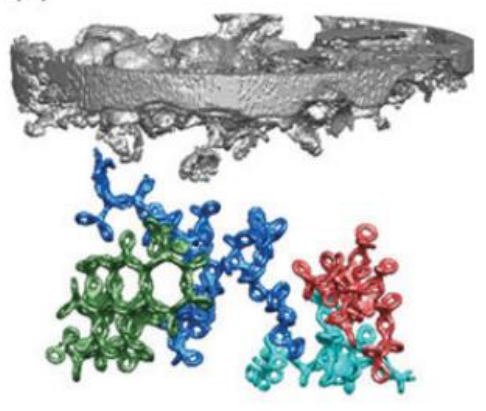

Figure 2: Progression of drying in a packed bed consisting of mono sized glass spheres visualized using X-ray tomography (Figure reported by Metzger, 2019 [4] reproduced with permission from Taylor \& Francis License number 5157130520121 obtained on 27/09/21)

\section{The Drying Curve}

Apart from presenting a good physical picture regarding the physics of the drying process, PNM studies also provide a very useful quantitative insight. Figure 3 shows what is referred to as a drying curve which is the rate of drying versus the pore saturation (fraction void filled). The dark area in the images denote the filled liquid and the light areas where liquid has evaporated. As can be seen on the right the network is complete saturated with the liquid with the highest evaporation rates. The evaporation rates remain constant until a point where it drops sharply. This is sharp drop is said to occur when the air network - left after the solvent evaporates, closes in on itself. This essentially forms a path of least resistance for air or a 'short circuit path' thereby limiting further evaporation rates. As can be seen at the point where the evaporation rate falls off, the saturation is still very high as shown in Figure 3 where inflection point coincides with a pore network liquid saturation fraction as high as $50 \%$.

This above finding is important as it may be connected to results obtained by Ottoboni et. al. $(2020)^{[1]}$. Shown in Figure 4 are the inlet and outlet temperature profiles obtained by Ottoboni et. al. $(2020)^{[1]}$ for varying inlet pressures and inlet setpoint temperatures. In the initial stages of drying, the intense vaporization of the solvent decreases the cake temperature substantially. As the drying air medium is in close contact with the cake, it may be assumed to be in thermal equilibrium with the air and its temperature drops too. Hence, the outlet temperature of the drying air medium decreases initially due to the transfer of the latent heat due to evaporation. During experiments, Ottoboni et. al. $(2020)^{[1]}$ detected an inflection point where the evaporation is no longer high enough to cause a reduction in the temperature. Hence, it was proposed as a criterion for dryland detection. 


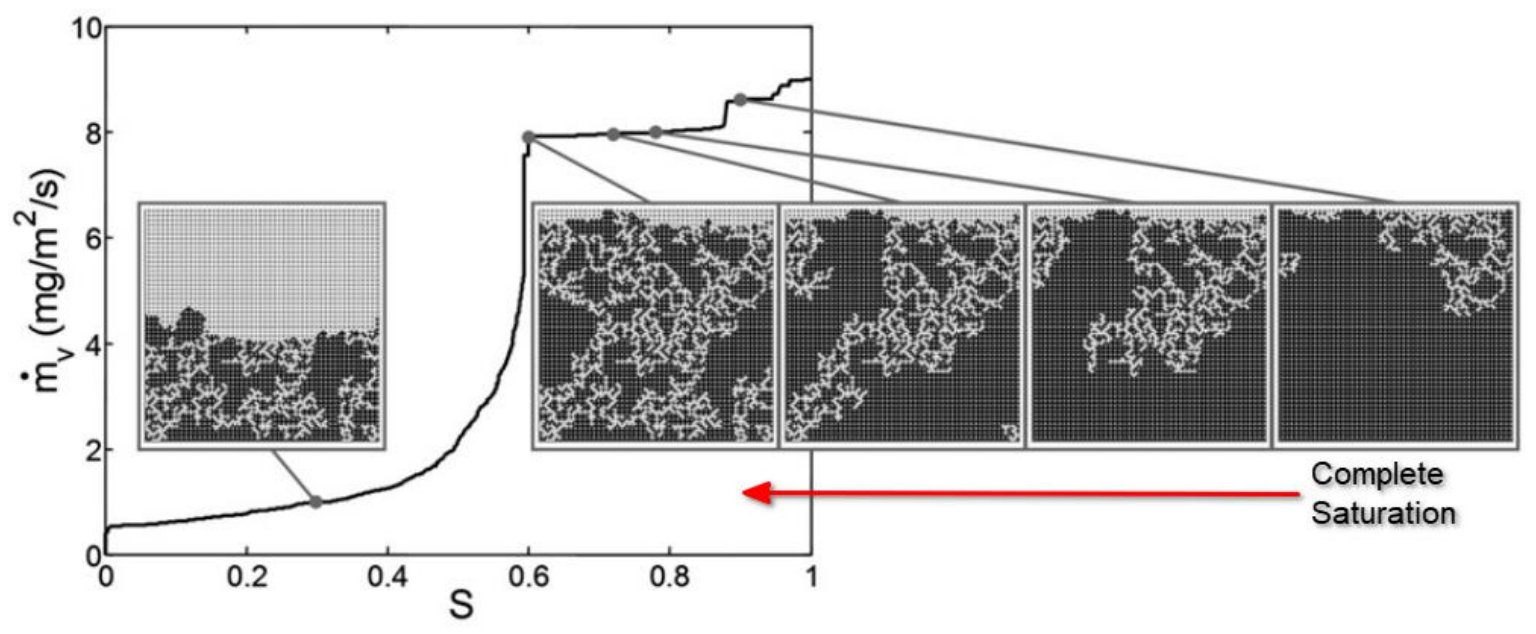

Figure 3: Rate of drying versus pore saturation and corresponding visual representation of pore network during the different stages of drying (Figure reported by Metzger, 2019 [4] reproduced with permission from reproduced with permission from Taylor \& Francis License number 5157130520121 obtained on 27/09/21 and John Wiley \& Sons License number 5114200448381 obtained on $22 / 07 / 21)$

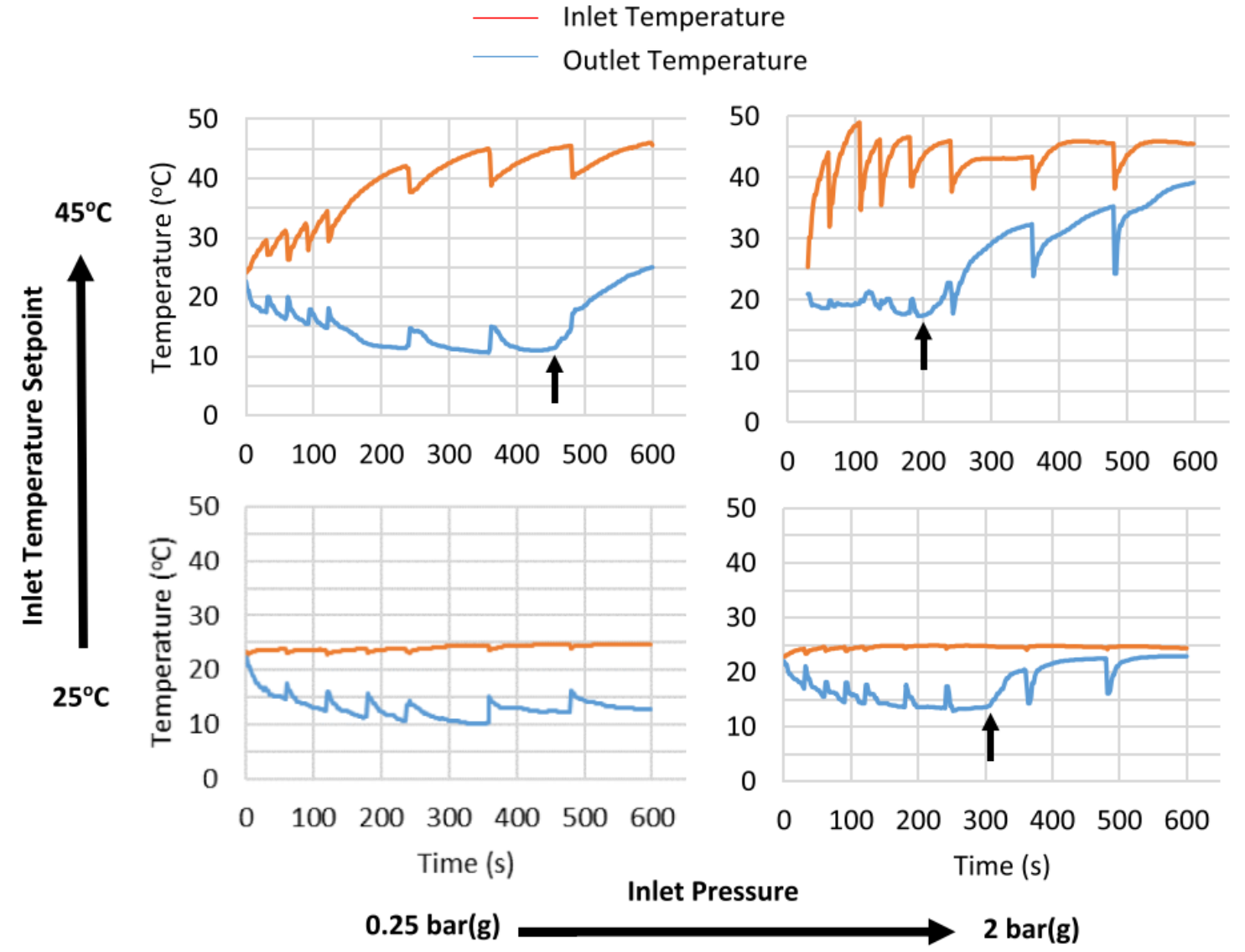

Figure 4: The inflection points for various operating conditions in the outlet temperature as a proposed criterion for dryland detection (Figure reported by Ottoboni et. al., $2020^{[1]}$ ) (Figure reproduced with permission from ACS on 21/07/21. Further permissions regarding the reuse of this material should be directed to the ACS) 
The inflection points in temperature shown in Figure 5 may immediately be connected to the representative drying curve shown in Figure 4 wherein, there is a sudden decrease in the vaporization rate in a pore network. As shown in Figure 4, corresponding to this sudden reduction in vaporization rate, the saturation in the cake would still be high (possibly as high as $50 \%$ ). Further, as this solvent is trapped for a substantial period, it would get saturated with the solids quickly as the dissolution time scale ( $\sim \mathrm{hr}$ ) is significantly lesser than the total drying time scale ( $24 \mathrm{hrs})$. Hence, it is expected that when the trapped solvent completely evaporates, it would lead to lump formation which was observed by Ottoboni et. al. (2020) ${ }^{[1]}$ as shown in Figure 1 to varying extents. Here it is important to keep in mind the caveats mentioned earlier that pore network studies consider stagnant air with only the top surface open. However, it may be argued that the fundamental nature of the drying rate curve, that a constant early period followed by a sharp drop and finally a long tail region, would be retained with different time scales.

\section{Possible Paths Forward}

The preceding analysis points to shortcomings of the conventional drying approaches but also identifies possible ways forward. Indeed, the liquid clustering problem may also be observed during the wash processes as under a pressure gradient any fluid will seek to find a path of least resistance referred to as the short circuiting of the flow. Additionally, for liquids there are surface tension effects which might be important. As seen in Figure 1, the problem was reported to be severe only for higher cake heights and to varying extents for smaller heights. However, this inevitably limits the scope for process optimisation and scale-up.

Typically drying of non-cohesive materials can be accomplished without any internals. However, powders belonging to Geldart C classifications or cohesive powders, require external vibrations, agitation, rotation, and centrifugation (Bait et. al., 2011) ${ }^{[6]}$. It may also be argued that the drying of other types of powders may also be intensified using the abovementioned operations. Based on the physical picture discussed in the present study, the solutions to the problem would need to be based on periodically breaking the pore network - which remains intact in the case of a settled cake throughout the process. In this authors view, this may be accomplished easiest, in terms of mechanical operation and continuous processing, by:

A. Adding an impeller in a vertical column which keeps stirring the solids at a slow speed throughout the duration of the drying process.

B. A vibrational bed under the solids which would keep disturbing the pore network - either in a column or as a separate moving bed 
Each option may have its own pros and cons. Option A is easier to implement but puts a limit on the shear, while Option B while being difficult to implement, has the added advantage of no shear limitation. A concept for a drying unit operation using an impeller (Option A) is shown in Figure 5A and for one using a vibrational bed (Option B) is shown in Figure 5B. For Figure 5A, the slurry enters a vertical chamber through the slurry port. The drying gas is then switched on after a given slurry volume is dispensed and the mother liquor is separated from the solids through pressure driven Nutsche filtration. During this time, the solids are stirred using an impeller which keep disrupting the pore network. After a suitable drying time, the solids may be removed from the bottom of the chamber by removing the movable filter media. The stirring and the drying gas may be kept operational to ensure the proper emptying of the chamber. A fluidic oscillator may be used to uniformly dispense the drying gas within the chamber ${ }^{[7]}$.

For Option B as shown in Figure 5B, a sieving tower may be modified by having the slurry enter through the top chamber. A drying gas may also be passed in a co-current fashion with a port available at the bottom separately to allow the gas to exit the tower. The constant vibration of the tower will ensure adequate separation of the particles potentially accomplished good drying conditions. The sieves may be selected to obtain particles within a specific range of particle sizes. The mother liquor may be collected in the bottom pan of the tower and recovered appropriately, and the solids may be collected in the sieve pan just above the bottom pan. An incline may be added in the solid collection sieve to leverage the vibrational motion and enable the solids removal in a continuous manner.

An impeller is typically used for powder blending or for the drying of adhesive/sticky particles. Vanarase et. al. (2011) ${ }^{[8]}$ performed experiments to identify the optimum rotation speed, weir angle, flow rate and blade configuration of a powder mixer for blending API powders having two different sizes. Toson et. al. (2018) ${ }^{[9]}$ performed the discrete element method simulations to characterize impact of operating parameters on the mixer performance for various particle sizes. The study consisted of a vertical mixer including key particle level properties such as particle velocities and particle residence times. Hoffman et. al. (2017) ${ }^{[10]}$ investigated the potential of a wire mesh for improving the drying efficiency of sticky particles simulated using ion exchange resins. Bait et. al. (2011) ${ }^{[8]}$ investigated various types of agitators and the impact of operating parameters such as inlet air velocity, temperature, agitation speed and feed loading on the drying kinetics in a batch mode fashion. Ribbon type agitators were found to perform the best while also being better from a scale-up point of view. 
(A)

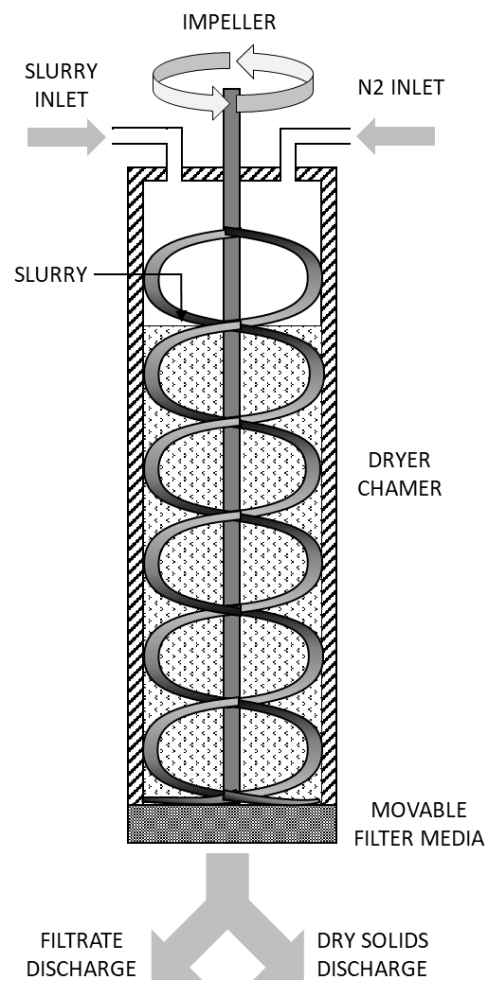

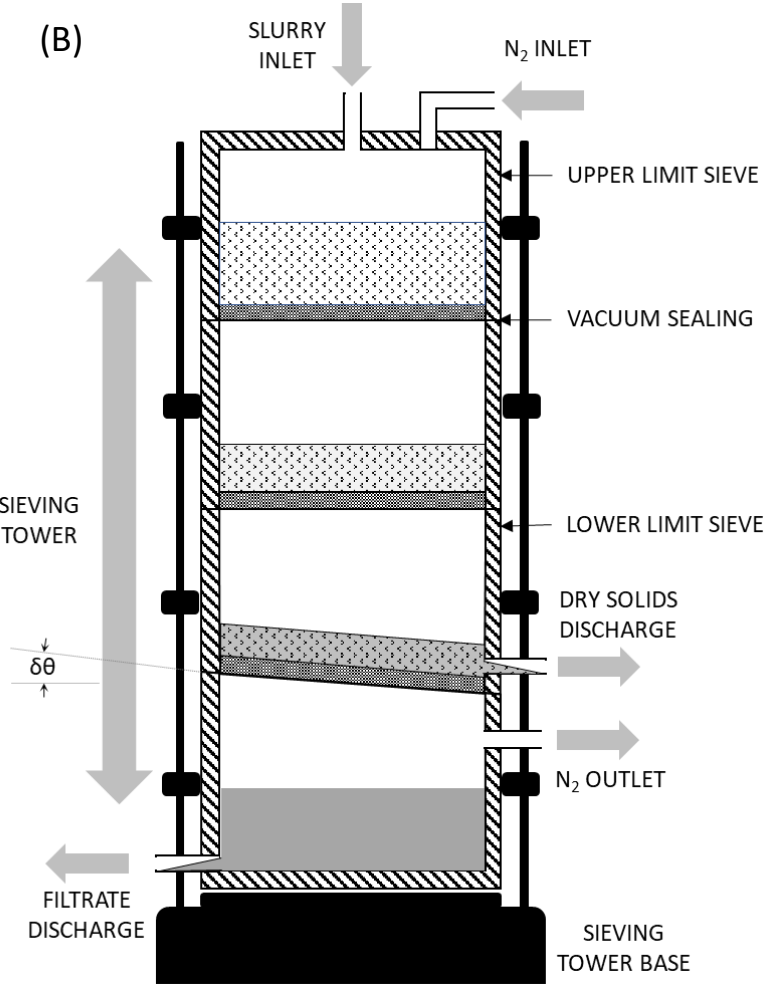

Figure 5: Schematic of prototypes for continuous drying (A) with impeller in a vertical chamber (B) using a retrofitted sieving tower as a vibrational bed

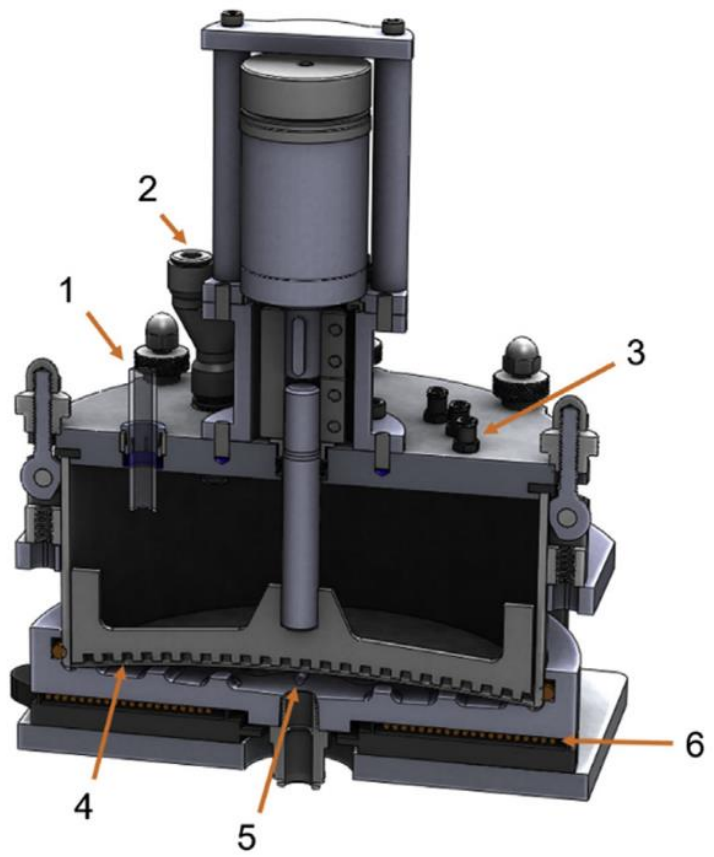

Figure 6: Cross sectional view of the filtration device reported by the MIT group (1) Feed suspension port (2) Compressed air/vacuum port (3) Wash solvent ports (4) $0.2 \mu \mathrm{m}$ filter mesh (5)

Thermocouple/Thermistor (6) Inductive heating plate (Figure reported by Capellades et. al., 2020 ${ }^{[3]}$ and reproduced with permission under Elsevier License number 5113700706913 obtained on 
It should be noted that Option A was implemented recently by Capellades et. al. (2020) for the case of continuous drying as shown in Figure 6 which allowed the processing of high filtration volumes (up to $4 \mathrm{~L}$ at a time) using relatively low shear ( 30 RPM) mechanical processing ${ }^{[3]}$. The caveat for this prototype was that the prototype consists of multiple semi-batch units which require manual intervention to switch during continuous operation and subsequent emptying/cleaning. One potential advantage of the Continuous Filter Dryer (CFD) used by Ottoboni et. al. (2020) is that by design, it processes small volumes - hence breaking the pore networks could be more efficient ${ }^{[1]}$. Further, if either option A or B is implemented, it is possible to do away with the washing stages entirely and just have a combined filtering-deliquoring stage. These solutions would remove the possible hurdles to process optimization, scale up.

\section{Conclusions}

The continuous drying of solids products is a key bottleneck in the continuous manufacturing of API products. In the present study, the problem is briefly discussed, and parallels are drawn to the pore network models typically used extensively to investigate the drying of porous solids. The proposed physical picture seems to answer why a solid cake may be significantly saturated even after the inflection point criterion for detecting the completion of drying as reported by Ottoboni et. al. (2020) is met ${ }^{[1]}$. Two possible setups are described which could potentially mitigate the fundamental challenges with drying and were compared existing prototypes reported by Capellades et. al. $(2020)^{[3]}$ and Ottoboni et. al. (2020) ${ }^{[1]}$. The proposed process modifications may potentially alleviate or entirely remove the bottleneck in continuous processing with respect to filtration and drying.

\section{Acknowledgements}

The authors would like to acknowledge the PROCESS COFUND fellowship which was co-sponsored by the Bernal Institute at the University of Limerick and Marie Skłodowska Curie Actions for enabling this research. The authors would also like to thank Professor Vivek Ranade for various inputs while preparing the manuscript.

\section{References}

[1] Ottoboni, S., Coleman, S. J., Steven, C., Siddique, M., Fraissinet, M., Joannes, M., Laux, A., Barton, A., Firth, P., Price, C. J., \& Mulheran, P. A. (2020). Understanding API static drying with hot gas flow: Design and test of a drying rig prototype and drying modeling development. 
Organic Process Research and Development, 24(11), 2505-2520.

https://doi.org/10.1021/acs.oprd.0c00035

[2] Domokos, A., Nagy, B., Gyürkés, M., Farkas, A., Tacsi, K., Pataki, H., Liu, Y. C., Balogh, A., Firth, P., Szilágyi, B., Marosi, G., Nagy, Z. K., \& Nagy, Z. K. (2020). End-to-end continuous manufacturing of conventional compressed tablets: From flow synthesis to tableting through integrated crystallization and filtration. International Journal of Pharmaceutics, 581(March), 119297. https://doi.org/10.1016/j.ijpharm.2020.119297

[3] Capellades, G., Neurohr, C., Azad, M., Brancazio, D., Rapp, K., Hammersmith, G., \& Myerson, A. S. (2020). A Compact Device for the Integrated Filtration, Drying, and Mechanical Processing of Active Pharmaceutical Ingredients. Journal of Pharmaceutical Sciences, 109(3), 1365-1372. https://doi.org/10.1016/j.xphs.2019.12.011

[4] Metzger, T. (2019). A personal view on pore network models in drying technology. Drying Technology, 37(5), 497-512. https://doi.org/10.1080/07373937.2018.1512502

[5] Debaste, F., \& Halloin, V. (2010). Application of discrete modeling approach to fluidized bed yeast drying. Journal of Food Process Engineering, 33(SUPPL. 1), 2-22.

\section{https://doi.org/10.1111/j.1745-4530.2008.00269.x}

[6] Bait, R. G., Pawar, S. B., Banerjee, A. N., Mujumdar, A. S., \& Thorat, B. N. (2011). Mechanically Agitated Fluidized Bed Drying of Cohesive Particles at Low Air Velocity. Drying Technology, 29(7), 808-818. https://doi.org/10.1080/07373937.2010.541574

[7] Khalde, C.M., Pandit, A.V., Sangwai, J.S. and Ranade, V.V., 2019. Flow, mixing, and heat transfer in fluidic oscillators. The Canadian Journal of Chemical Engineering, 97(2), pp.542-559. https://doi.org/10.1002/cjce.23377

[8] Vanarase, A. U., \& Muzzio, F. J. (2011). Effect of operating conditions and design parameters in a continuous powder mixer. Powder Technology, 208(1), 26-36.

https://doi.org/10.1016/J.POWTEC.2010.11.038

[9] Toson, P., Siegmann, E., Trogrlic, M., Kureck, H., Khinast, J., Jajcevic, D., Doshi, P., Blackwood, D., Bonnassieux, A., Daugherity, P. D., \& am Ende, M. T. (2018). Detailed modeling and process design of an advanced continuous powder mixer. International Journal of Pharmaceutics, 552(1-2), 288-300. https://doi.org/10.1016/J.IJPHARM.2018.09.032

[10] Hoffman, P., Pěnička, M., \& Fořt, I. (2017). Effect of Fluidized Bed Stirring on Drying Process of Adhesive Particles. Chem. Biochem. Eng. Q, 31(1), 1-10.

https://doi.org/10.15255/CABEQ.2015.2335 